\title{
Aproximación crítica a los cursos de lingüística en la Universidad $^{1}$
}

\author{
Juan Camilo Puentes Castro \\ Universidad Surcolombiana, Colombia \\ JuanPuentesCastro@gmail.com
}

\section{Resumen}

Este trabajo se sitúa en la relación Saber disciplinar - Saber pedagógico, el propósito es responder la pregunta ¿cómo se evidencia el Pensamiento Crítico en lo que se refiere al componente específico de Lingüística con miras al perfil profesional del licenciado? Para ello se reflexiona sobre el objeto de estudio de la lingüística, su praxis como ciencia y su papel en la pedagogía de los idiomas.

Palabras claves: Pensamiento crítico, aprendizaje significativo, lingüística, saber disciplinar, saber pedagógico.

\section{Critical approach to linguistics courses at the University}

\begin{abstract}
This work is about the relation between Pedagogical knowledge and disciplinary knowledge, in order to know how the Critical Thought is demonstrated regarding the specific component of Linguistics, with a view to the professional licentiate profile. For that reason it's important to reflect about the object of the linguistic studies, the praxis as a science and its pedagogic role into the languages.
\end{abstract}

Keywords: critical thought, significant learning, linguistic, Pedagogical knowledge and disciplinary knowledge

1 Esta ponencia es resultado del proyecto de investigación del Semillero SOKAL titulado "Aproximación critica a los cursos de lingüística en la universidad: una visión desde el pensamiento crítico y el aprendizaje significativo" financiado por la Vicerrectoría de Investigación y Proyección Social y por el grupo de investigación IPPE de la Universidad Surcolombiana. En la investigación participaron los colegas Anthony Forero, Karla Ibáñez, Alexandra González y se contó con la orientación del profesor Miguel Ángel Mahecha Bermúdez, docente del área de Lingüística de la Facultad de Educación. 


\section{Introducción}

En el año 2007, cuando el Ministerio de Educación Nacional (MEN) expidió la resolución número 4627 "por medio de la cual se le otorga la acreditación al programa de Lingüística de la Universidad Nacional", uno de los puntos que más llamó la atención fue una de las recomendaciones dadas por el MEN, la cual invita al programa a "considerar en correspondencia con el perfil del egresado la distribución y orientación de las asignaturas y analizar la posibilidad de fundamentar un perfil para la docencia, dado que muchos egresados tienen desempeño como docentes".

Esto llevo a que se pusiera, de nuevo, sobre la mesa la relación existente entre lingüística y educación. Un tema abordado dúrate la década de los ochenta y noventa por personajes como el maestro Luis Ángel Baena quien es fundamental a la hora de comprender esta relación. El enfoque semántico comunicativo, desarrollado por este autor, pareció, en su momento, la salida más diplomática a este debate, razón por la que el MEN decide usarlo, mal parafraseado, como base teórica para la formación de los estándares para el área de Lengua Castellana. Pero luego de este suceso, la relación dicotómica entre la ciencia y este campo de aplicación es tema de controversia y es necesario re-pensarlo desde las diferentes áreas que hacen uso del corpus conceptual de la lingüística, partiendo, obviamente, desde el departamento de Lingüística de la UN.

Recientemente hemos visto que "las diversas tendencias que se manifiestan en el estudio del lenguaje han producido resultados de valor indiscutible, pero distan mucho de haber creado un cuerpo de doctrina en el cual pueda resumirse la vigencia y durabilidad exigibles a los conocimientos que deben transmitirse en los grados primario y segundario de la educación"(Mora, 1988), esta situación se presenta tanto en la escuela, como a la hora de enseñar lingüística, es decir en la relación Educación-Lingüística. Por esto, a la hora de pensar la relación Lingüística-Educación es necesario revisar primero la relación Educación-
Lingüística, saber ¿cómo se está enseñando la lingüística? ¿Qué tipo de tópicos son pertinentes para la formación en lenguaje? para así luego entender, ¿qué elementos del corpus lingüístico pueden aportar para la formación en pedagogía de los idiomas?

Surge en esta primera etapa de la indagación un problema esencial pues "el área de lingüística debe cubrir necesidades de aplicación para estudiantes de terapia del lenguaje (fonética y fonología, psicolingüística, sintaxis), para estudiantes de filosofía (pragmátic a, argumentación, análisis del discurso, epistemología lingüística), para los antropólogos (etnolingüística, sociolingüística, etnografía del habla y de la comunicación) [...]" (Mahecha, 2009) lo que lleva a concluir que los contenidos de la ciencia que deben enseñarse depende de su campo de aplicación, es decir que un curso de lingüística para lingüistas es diferente a un curso de para licenciados en pedagogía de los idiomas.

Pero pensar en los diversos campos de aplicación del corpus teórico nos obliga necesariamente a pensar en lo planteado por Saussure en sus Escritos de lingüística General ¿Cuál es el objeto a la vez integral y concreto de la lingüística? La cuesti ón es particularmente difícil; otras ciencias operan con objetos dados de antemano y que se pueden considerar en seguida desde diferentes puntos de vista. No es así en la lingüística, por eso lejos de preceder el objeto al punto de vista, se diría que es el punto de vista el que crea el objeto, $y$, además, nada nos dice de antemano que una de esas maneras de considerar el hecho en cuestión sea anterior o superior a las otras. Así, pues, de cualquier lado que se mire la cuestión, en ninguna parte se nos ofrece entero el objeto de estudio (Saussure, 2004).

Este tema ha sido un problema para la ciencia, primero por los diversos problemas tanto disciplinares como extra-disciplinares que se han presentado, y segundo porque ha dificultado poder comprender el uso de la lingüística, sus impactos, su aplicación. Por ejemplo en la relación Lingüística-Educación el trabajo se ha limitado al enfoque comunicativo, lingüística 
del texto y análisis del discurso, enfoques que se acercan a lo que el ministerio plantea como fin último de la enseña de lengua materna: el desarrollo de competencia comunicativa.

Pero esta distinción deja por fuera otros elementos fundamentales a la hora de comprender el trabajo con lo comunicativo, dado que si partimos de las tres funciones, que según Halladay, tiene el lenguaje a saber: a. Una función ideativa o conceptual de carácter Intra-individual (lenguaje), b. Una función interaccional o comunicativa de carácter interindividual (lengua), c. Una función textual (Habla); se estaría centrando el trabajo solo en la última, que en realidad no sería una función como lo son las dos primeras, pues esta posee características de rasgo funcional.

Esto quiere decir que no partimos del significar como proceso de producción del sentido, sino desde los significados de los discursos (textos) ${ }^{2}$ como "utilización del lenguaje en la recreación del sentido de nuestra experiencia del mundo". Olvidándonos del proceso Intra-idividual "que se cumple en la utilización del lenguaje en la transformación de la experiencia humana de la realidad objetiva natural y social en sentido", y del proceso Inter-individual "que se realiza en la utilización del lenguaje como instrumento de la interacción humana", lo que reduce la enseñanza de los idiomas a su mera expresión empírica.

Este enfoque planteado por el profesor Baena parte de la idea de considerar para la relación Lingüística-Educación el significar como objeto de estudio, según Mahecha podría decirse que el objeto del lingüista son las unidades que generan sentido (Mahecha, 2012, p. 24), y los procesos para generar ese sentido. Siguiendo este planteamiento podemos afirmar que en esta relación hay como fin último: "el análisis de lo que significa significar en la práctica discursiva, vista como la práctica de la utilización del lenguaje como instrumento que realiza la semantización de la totalidad de la experiencia humana en relación con la realidad objetiva, natural y social" (Baena, 1989).

Por eso, por ejemplo en el caso de la formación en lingüística para pedagogía de los idiomas se deba comprender las diversas ramas de estudio de la lingüística, comprender los objetos en su totalidad, lo que generó la pregunta de investigación: ¿cómo se evidencia el Pensamiento Crítico en lo que se refiere al componente específico de Lingüística con miras al perfil profesional del Licenciado en lengua materna?

\section{Marco Teórico}

Para formular respuesta a esta pregunta de investigación hay que definir cuál es la relación entre saber disciplinar y saber pedagógico que vamos a tomar, pues en esta propuesta se reevalúa la relación en la que la pedagogía surge de querer hacer digerible un conocimiento teórico que pertenece a la órbita de los discursos disciplinares. por el contario y siguiendo a Grossman hay que decir que cada disciplina funda y crea su propia pedagogía generada a partir de sus métodos de trabajo, su desarrollo, su concepción epistémica, etcétera. Pamela Grossman depuro la propuesta realizada por Shulman, y en su concepción las categorías de los saberes necesarios para acercarse a la docencia son cuatro: el conocimiento pedagógico general, el conocimiento del contenido, el conocimiento pedagógico del contenido y el conocimiento del contexto.

Es decir que la relación saber disciplinar y saber pedagógico se apoya en el conocimiento pedagógico del contenido. Pues las conexiones que se establecen entre el conocimiento sobre pedagogía y el conocimiento de la disciplina que tiene el docente, permite la transformación del contenido para su enseñanza. Esto se debe a que la pedagogía es un recurso que surge en el

2 Reflexione sobre los tipos de preguntas que se hacen en las pruebas llevadas a cabo por el ICFES, siempre acuden al significado no a la significación. 
camino recorrido por esos linderos disciplinares, pero siempre pensando que debe existir una conexión, unas relaciones en cada uno de los elementos, unos criterios que orienten la subjetividad para no caer en caprichos conceptuales. Entonces, lo que pretende la indagación es comprender si ese contenido teórico desarrollado en los cursos de lingüística tiene algo de significativo para los estudiantes (Aprendizaje Significativo), cómo se concibe la relación Lingüística-Educación (pensamiento crítico) y cómo se concibe la tarea docente desde el lenguaje.

Para comprender si los estudiantes tienen claro nociones básicas del corpus conceptual, se tomaron las tres funciones del lenguaje planteadas por Halladay, y se asocio la primera al lenguaje, la segunda a la lengua y la tercera al habla; entendiendo que al comprender la asociación entre estos conceptos, por ende de sus funciones, se está dando cuenta de las dimensiones estudiadas por la ciencia de la significación. Lo que se pretende es tomar al hablante, aquel que produce discursos (textos), como ser humano y como ser social que por medio del proceso de la significación usa el lenguaje como instrumento de la semantización de la totalidad de la experiencia humana, esto nos acerca al preciso papel del lenguaje en el proceso de conocimiento, así como lo plantea Baena; es decir que el conocimiento, asociación y distinción de estos conceptos es necesario para cualquiera que quiera comprender lingüística, por eso se indaga sobre la forma en la que se conciben, es decir el nivel de pensamiento crítico y apropiación del corpus conceptual de la ciencia ${ }^{3}$.

Ahora, sobre el P.C se ha dicho mucho y cada intento de definición lo que hace es complicar aún más la posibilidad de llegar a algún consenso, por eso para definirlo partimos de un trabajo realizado por Marciales (2003), en el que se encuentra una larga disertación sobre lo que se ha entendido por P.C. Para este trabajo se le han agregado otros elementos, a saber:

El pensamiento crítico que indagamos en este trabajo responde a "la formulación de juicios razonados, bien sea sobre aspectos de orden escolar, familiar, laboral, o sobre otros que demanden el ejercicio de tales capacidades humanas. El ejercicio del pensamiento crítico supone valorar desde criterios razonables y tomando en cuenta los contextos en los cuales tales valoraciones son formuladas. Pensar críticamente supone contar con criterios que guíen el proceso" (Santiuste, et al, citado por Marciales, 2003, p. 60). La generación de esos criterios, característica fundamental del pensador crítico son generadas por unas estrategias, que son:

Cuadro 1. las estrategias genéricas para ser un pensador critico (Marciales, 2003: 59-60)

\begin{tabular}{|c|c|}
\hline $\begin{array}{l}\text { ESTRATEGIAS } \\
\text { PARA SER UN } \\
\text { PENSADOR } \\
\text { CRÍTICO }\end{array}$ & DEFINICIÓN \\
\hline $\begin{array}{c}\text { EL PENSAMIENTO } \\
\text { CRÍTICO SE BASA } \\
\text { EN CRITERIOS }\end{array}$ & $\begin{array}{l}\text { Un criterio es entendido como la regla o principio utilizado en la } \\
\text { realización de juicios, que son emitidos por criterios bien definidos. Los } \\
\text { criterios son razones que deben tener un alto grado de aceptabilidad. El } \\
\text { uso competente de estas razones establece la objetividad de los juicios. }\end{array}$ \\
\hline $\begin{array}{l}\text { METACRITERIOS Y } \\
\text { MEGACRITERIOS }\end{array}$ & $\begin{array}{l}\text { La elección de un criterio obedece a otros criterios, los metacriterios, } \\
\text { que dan cuenta de la coherencia, la consistencia, la veracidad, la fuerza y la } \\
\text { pertinencia de los primeros. Algunos otros son de un nivel de generalidad } \\
\text { muy elevados: verdad, lo correcto, lo no correcto, lo bueno, lo bello. }\end{array}$ \\
\hline $\begin{array}{l}\text { EL PENSAMIENTO } \\
\text { CRÍTICO ES } \\
\text { AUTOCORRECTIVO }\end{array}$ & $\begin{array}{l}\text { Es un pensamiento que se observa a sí mismo, que no es lo mismo que } \\
\text { afirmar que todo proceso metacognitivo sea equivalente a un pensamiento } \\
\text { crítico. En la medida en que cada participante es capaz de interiorizar de } \\
\text { forma global la metodología de la comunidad, será capaz de aplicar procesos } \\
\text { autocorrectivos a su propio pensamiento. }\end{array}$ \\
\hline $\begin{array}{l}\text { EL PENSAMIENTO } \\
\text { CRÍTICO } \\
\text { DESARROLLA } \\
\text { SENSIBILIDAD AL } \\
\text { CONTEXTO }\end{array}$ & $\begin{array}{l}\text { Implica el reconocimiento de circunstancias excepcionales o irregulares, } \\
\text { limitaciones especiales, contingencias o constricciones, configuraciones } \\
\text { globales, evidencias insuficientes y da la posibilidad de que algunos } \\
\text { significados no puedan traducirse de un contexto a otro. }\end{array}$ \\
\hline
\end{tabular}

Estas estrategias, son el conjunto de procedimientos de los cuales dispone el sujeto para operar sobre los conocimientos que posee y aquellos nuevos; pero estas por si solas no logran nada, hay que tener un dominio sobre el contexto del sujeto que le exige responder de manera

3 Esta distinción entre lenguaje, lengua y habla puede ser usada para evaluar cualquier curso de lingüística, en cualquiera de sus ramas aplicativas, pues nos genera la necesidad de comprender varios elementos tanto de la historia de las ideas lingüísticas, como de sus áreas de influencia. Otra de las preguntas que se pueden formular es ¿en cuál de esos tres niveles se sitúa el estudio realizado por determinada carrera, por ejemplo la terapia del lenguaje? y ¿cómo se relaciona este lugar de enunciación con los demás conceptos? 
razonada y coherente con la situación y por último unas motivaciones (trabajadas desde el P.C) que hacen referencia al vínculo que establece el sujeto con el conocimiento, ese vínculo afectivo que mueve su curiosidad e invita al desarrollo de una actitud positiva frente al conocer, lo que se entiende por Aprendizaje Significativo, gráficamente representado así: (Santiuste et al, citado por Marciales, 2003: 61)

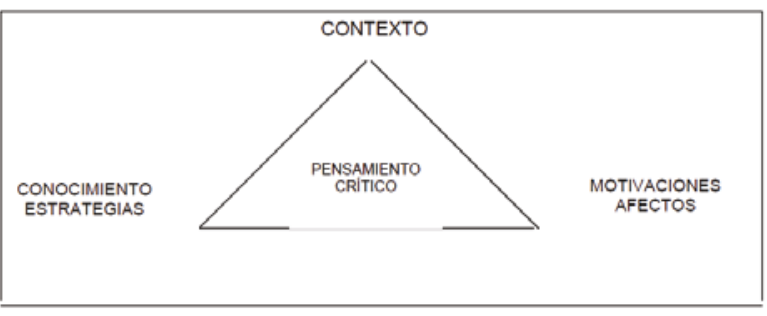

Figura 1. Tomada de Santiuste 2001 (Marciales, 2003: 62)

Estos tres elementos permiten construir las categorías de análisis a utilizar para poder caracterizar el P.C con referencia al dominio disciplinar llevado a cabo por los estudiantes a partir de la significación que le dan al discurso disciplinar, con miras al perfil profesional del licenciado:

Categoría de análisis 1: Manejo de Conceptos

- Conocimiento disciplinar del área (corpus conceptual de la ciencia): la distinción y relación entre lenguaje, lengua y habla.

- Relación de las diferentes escuelas lingüísticas con los conceptos de Lenguaje, Lengua y Habla

- Conocimiento de la historia de las ideas lingüísticas y sus respectivas tesis.

- Interés conceptual de cada modelo: asociado a algunas de las tres dimensiones estudiadas.

- Bibliografía básica.

- Composición de cada modelo y campo de aplicación de sus aportes.

\section{Categoría de análisis 2: Uso práctico del conocimiento} teórico

- Contextualización de la teoría en la enseñanza de lengua materna: cómo se insertan los conceptos Lenguaje, Lengua y Habla en los diferentes contenidos ensañados en la escuela.

- La relación entre pedagogía (como ciencia para el conocimiento) y la Lingüística (como ciencia que estudia el lenguaje y su influencia en la experiencia del conocer).

- Articulación de la información con las necesidades aplicativas.

Categoría de análisis 3: Coherencia y sentido

- No arbitrariedad y sustantividad.

- Información asimilada y significativa para el estudiante, partiendo de su experiencia y sus criterios de posterior aplicación.

- Valor dado a la información interiorizada a partir de su relevancia para el estudiante (Valor social del saber).

- Interacción que se da entre lo que subyace a cada saber (es decir, a su epistemología) y los puntos de vista que ofrecen sus actores, evidenciando con ello la manera en que la persona actúa sobre su saber previo.

- La acomodación de la información (nueva y vieja) en el interior de la estructura cognitiva.

\section{Categoría de análisis 4: Memorización}

- La fijación que tiene la información en la estructura cognitiva, que se hace útil ante determinada situación.

- Memorización subordinada: el afianzamiento de la información solo es posible siempre y cuando la información que se recibe corrobora lo que ya sabe o modifica, califica y extiende la información sabida.

- Memorización superordenada: aquí el aprendizaje tiene en cuenta que el aseguramiento de la información se va dando de acuerdo a su importancia. De ahí que las informaciones se organicen jerárquicamente en la memoria.

- Memorización combinatoria: la memoria se consolide teniendo en cuenta la importancia y cambios que se presentan, pero con la salvedad de que las informaciones buscan ofrecer imágenes genéricas y relevantes sobre lo que se ha asimilado.

- La memoria es lo que posibilita que se dé la asimilación.

\section{Materiales y Métodos}

Para poner esto en marcha se decidió primero, realizar una revisión de los microdiseños curriculares de los seis cursos que componen el área de lingüística, de ellos se revisaron los 
objetivos generales, las justificaciones y las habilidades a desarrollar en cada uno, pero de estas habilidades que se configuran en el Saber, Saber-Hacer y el Ser solo tomamos las dos primeras, pues hay que entender que la formación del Ser queda en manos e interés personal del docente y de sus discursos ${ }^{4}$.

La razón por la que se decidió empezar por aquí fue porque estos documentos nos permiten conocer cómo se tiene planeado el desarrollo de la línea y partiendo de ahí se puede conocer lo que se considera necesario dominar del área en un curso. Lo que se analizó de estos documentos hace referencia al proceso entre Saber disciplinar y saber pedagógico, por eso se reflexiona sobre la contextualización de la teoría en la enseñanza de lengua materna, la relación entre pedagogía (como ciencia para el conocimiento) y la Lingüística (como ciencia que estudia el lenguaje y su influencia en la experiencia del conocer), y la articulación de la información con las necesidades aplicativas como licenciados en pedagogía de los idiomas.

Luego se construyó el siguiente instrumento que partió de la experiencia desarrollada en la UPTC, por los docentes Joselyn Corredor Tapias y Cesar Romero Farfán, quienes diseñaron una prueba cerrada para evaluar dos grupos de un curso de Lingüística General de la carrera de Idiomas Modernos, durante el primer semestre del 2009. Esta experiencia fue importante para el desarrollo de este trabajo, pero con respecto a esa prueba esta pretende ser menos mecánica y limitada.

\section{UNIVERSIDAD SURCOLOMBIANA - FALCULTAD DE EDUCACIÓN Realizado: Semillero SOKAL Código:}

Nombre:

Determinar el objeto de estudio de la lingüística es bastante difícil, ya lo plantea F. de Saussure en el Curso de Lingüística General: "Cuál es el objeto a la vez integral y concreto de la lingüística? La cuestión es particularmente difícil; [...] Otras ciencias operan con objetos dados de antemano y que se pueden considerar en seguida desde diferentes puntos de vista. No es así en la lingüística. [...] Lejos de preceder el objeto al punto de vista, se diría que es el punto de vista el que crea el objeto, y, además, nada nos dice de antemano que una de esas maneras de considerar el hecho en cuestión sea anterior o superior a las otras. [...] Así, pues, de cualquier lado que se mire la cuestión, en ninguna parte se nos ofrece entero el objeto de la lingüística". A partir de lo anterior, contemplemos diferentes visiones, planteamientos de ese objeto de estudio:

"A nuestro parecer, no hay más que una solución para todas estas dificultades: hay que colocarse desde el primer momento en el terreno de la lengua y tomarla como norma de todas las otras manifestaciones del lenguaje. En efecto, entre tantas dualidades, la lengua parece ser lo único susceptible de definición autónoma [...]" (SAUSSURE, 1916. PÁG. 33)

"La lingüística se ocupa del fenómeno que constituye el lenguaje y, por supuesto, sin descuidar la porción del lenguaje que s e transforma en texto [...] la lingüistica, por su parte -y es lo que la diferencia de toda otra disciplina científica-, se ocupa de una cosa que no es objeto ni sustancial en el lenguaje, ¿qué es lo que hay? Los datos del lenguaje no existen sino por sus diferencias, no valen más que por sus oposiciones". (BENVENISTE, 1979, pág. $32,34)$

"[La Lingüística] es la ciencia que estudia desde todos los puntos de vista posibles el lenguaje humano articulado, en general y en las formas específicas en que se realiza, es decir, en los actos lingüísticos y en los sistemas de isoglosas que, tradicionalmente o por convención, se llaman lenguas". (COSERIU, 1983, pág. 7)

"[...] El lingüista indaga sobre el hablar, sobre el significar. Podría decirse entonces que el objeto del lingüista son las unidades que generan sentido" (MAHECHA, 2011, pág. 20)

"La razón para concentrarse en el estudio de los actos de habla es, simplemente, esta: toda comunicación lingüística incluye actos lingüísticos. La unidad de la comunicación lingüística no es, como se ha supuesto generalmente, el símbolo, palabra, oración [...] sino más bien la producción o emisión del símbolo, palabra u oración al realizar el acto de habla. Considerar una instancia como un mensaje es considerarla

4 Esta es otra línea interesante que se encontró en este trabajo ¿Qué tipo de profesional proyectan los docentes a partir de sus discursos disciplinares? 
como instancia producida y emitida. Más precisamente, la producción o emisión de una oración- instancia bajo ciertas condiciones constituyen un acto de habla y los actos de habla [...] son las unidades básicas o mínimas de la comunicación lingüística". (SEARLE, pág. 26)

"[...] La diversidad de la conducta humana, su adecuación a situaciones nuevas y la capacidad del hombre para innovar -el aspecto creador del uso del lenguaje proporciona la indicación principal de esto- [...] de este modo, una psicología totalmente adecuada requiere la postulación de un "principio creador» junto con el «principio mecánico»" (CHOMSKY, 1974, pág. 25)

1. “Según Morales (1995, pp. 119-120) [Apoyado en Apresian dice]: un modelo no es más que un dispositivo teórico mediante el cual se logra progresivamente un dominio conceptual de la realidad. Siempre se construye un modelo de un objeto para lograr un conocimiento más profundo de dicho objeto [...] el modelo construido no entra en conflicto con lo que representa $[\ldots] "$.. Seleccione un modelo teórico del siglo XX, el cual esté relacionado con una de las anteriores citas e identifique su autor (datos biográficos), tesis, argumentos, conclusiones, bibliografía (textos fundamentales).

2. Seleccione tres de las anteriores citas e ilustre la pertinencia de los objetos de estudio (modelos), teniendo en cuenta el perfil profesional del programa de LC.

3. Seleccione la (s) cita (s) que considera le permiten establecer (hacer explícito) una relación y distinción de los conceptos de lenguaje, lengua y habla.

4. Redacte un texto en donde identifique (distinga) la idea central que atraviesa todos los documentos.

La población a la que se le aplicó fueron los estudiantes del programa de Lengua castellana de la USCO, durante el primer semestre de 2012, para la prueba la población fue dividida en dos grupos, un primer grupo conformado por los estudiantes del curso Lingüística I, ya que ellos estaban iniciando un acercamiento a los temas lingüísticos, pues hasta el momento solo contaban con la aprobación de un curso introductorio, en donde se trabaja de manera general la historia de las ideas lingüísticas. El grupo dos estaba conformado por estudiantes de diferentes semestres, ya que estos poseían un conocimiento más sólido pues había tomado ya unos cursos de lingüística, e incluso algunos ya estaban realizando la práctica pedagógica. Es por eso que esta prueba fue elaborada teniendo en cuenta toda la población, razón por la que se toman los conceptos Lenguaje, Lengua y Habla, su definición, relación y distinción como norte del dominio disciplinar, y desde ahí poder identificar la evolución en la complejidad de los discursos desarrollados por los estudiantes, dependiendo de su nivel de avance en la carrera.

\section{Discusión}

Lo más destacable a la hora de enfrentarse a los microdiseños es que en cuanto a la revisión de los objetivos de cada curso podemos encontrar que cada uno tiene un fuerte interés por el conocimiento teórico-práctico del saber disciplinar, dejándolo manifiesto literalmente. El curso de introducción es el más amplio, pues busca mostrar todas las formas en las que el hombre ha reflexionado sobre el lenguaje y la composición de esta como ciencia, otro elemento importante en los cursos es el análisis formal de las unidades lingüísticas en cuanto a su forma y función, luego podemos ver que del curso I al V cada uno tiene interés en ampliar cada una de las formas en las que se trabaja el lenguaje. De aquí se puede decir que no hay una jerarquización por grado de complejidad en los cursos, lo que traduce que estos se podrían tomar en desorden, y no afectaría en nada la formación del estudiante.

En cuanto a las justificaciones encontramos un interés real por la formación de un "nuevo" maestro de lengua castellana, aunque no queda claro cómo hacerlo. También hay un profundo interés en integrar las diferentes esferas sociales, en desarrollar el entendimiento de cómo se lleva a cabo las interacciones comunicativas, y comprender las dinámicas que se desarrollan en la sociedad y la cultura. De esta relación se puede entender la importancia del enfoque comunicativo como resultado de la interacción entre saber disciplinar y pedagógico, aunque se debe reconocer que se deja de lado otros elementos como lo cognitivo, lo biológico, olvidando que la lingüística es una ciencia de la significación.

Sobre las habilidades encontramos un interés por el desarrollo del conocimiento disciplinar que le ayude a dar cuenta, sobre 
todo, por la dimensión formal y comunicativa de lengua. Hay también un interés porque el estudiante reconozca las diversas posibilidades investigativas que tiene la lingüística para acercarse a un objeto determinado, hay que hacer énfasis especial en la habilidad que refiere a que desde el discurso científico se construyan propuestas didácticas para la comprensión de las competencias lingüística y comunicativa.

Ahora, con respecto a la prueba, la primera pregunta fue analizada a partir del Manejo de conceptos y la Memorización, pues para este punto se debía responder a datos precisos (Memorización) que se esperan estén interiorizados por los estudiantes; pues sin estos conocimientos memorísticos es difícil realizar un proceso de acomodación (Manejo de conceptos) de nueva información ya que no habrá conocimientos previos que sirvan de puente para su asimilación. Lo que se encontró fue un conocimiento muy escaso sobre autores paradigmáticos como Saussure o Chomsky y su que hacer disciplinar, aunque este último fue reconocido por algunos más por el activismo político; sobre los demás no hay referencias claras.

La segunda pregunta fue revisada a partir de la categoría Uso práctico del contenido puesto que para resolver esta pregunta es indispensable, además de conocer y entender los planteamientos que hacían los autores, tener claridad sobre lo que propone el perfil del licenciado en Lengua Castellana. Lo que se encontró es que los estudiantes desconocen para qué se están formando y no encuentran una relación entre los contenidos disciplinares y su ejercicio docente; de ahí que la Lingüística sea vista con poca utilidad.

La tercera pregunta fue revisada a partir de la categoría Coherencia y sentido y Manejo de conceptos pues esta encierra el conocimiento genérico que ha adquirido el estudiante durante su proceso de formación en el área de Lingüística y el sentido propio que se le da al campo disciplinar. Sin embargo, lo que se encontró es que los estudiantes no tienen claridad sobre estos conceptos y por lo tanto no lograron establecer un sentido jerarquizado a estos elementos, esto significa que no comprenden que la lingüística tiene diversas áreas de influencia más allá como lo mental-cognitivo y lo articulatorio, que son indispensables para comprender lo comunicativo.

La última pregunta es bastante compleja, pues se pretende darle libertad al estudiante de que aplique sus propios criterios para comprender la prueba. En este punto se requiere tanto de un dominio teórico como práctico, pues se debía tener claridad de lo que se planteaba en las anteriores preguntas para poder construir un texto coherente que rescatara la idea central. En esta pregunta los estudiantes en su mayoría elaboraron la respuesta, sin embargo esta no era coherente con lo que se les indicaba, sino que se limitaron a explicar o parafrasear las citas de los autores sin relacionar ese conocimiento teórico con lo práctico, por el contrario reflexionaron sobre el papel del docente de forma idealizada, como suele concebirse desde la universidad.

\section{Conclusión}

De lo anterior podemos concluir que no hay claridad a la hora de comprender la relación Educación-Lingüística, entiendo esta como la forma en la que se enseña lingüística; ni tampoco en la relación Lingüística-Educación, comprendiendo esta como los aportes que da la ciencia de la significación al procesos de enseña de las lenguas. Por esto los estudiantes aun no tienen claro hacia dónde va la formación en lingüística para estas carreras, por consiguiente sus niveles de aprendizaje significativo son muy bajos, razón por la que no hay una desarrollo mancomunado entre lo Significativo y lo Crítico, haciendo uso de este último como algo que nos auto-imponemos sin unos juicios claros, acudiendo solo intereses personales.

Esto también lo podemos evidenciar a la hora de evaluar los diversos cursos pues aun no hay claridad de ¿qué elementos del corpus debe dominar el licenciado en lenguas? razón por la que este documento intenta aportar en esa línea en la que el perfil del lingüista de la UN se cruza con el de los licenciados en idiomas, y comprender que el cambio que se pide a gritos desde las aulas universitarias está, en buena parte, en la comprensión de la influencia de la 
ciencia de la significación dado que la incursión del significar en la educación llevaría a que se desarrollará una formación ya no para el conocimiento, si no en el conocimiento.

Esto mismo es necesario para la enseñanza de la lingüística en las universidades,es por eso que surge la necesidad de pensar en la naturaleza y el interés especial de los cursos de Lingüística, partiendo de la praxis del corpus conceptual de esta para pensar en un "modelito" que permita articular los elementos que configuran la significación con miras a su trabajo en el desarrollo del conocimiento, para ello es necesario integrar lo biológico y lo social con miras al desarrollo del conocimiento, repensando el modelo desarrollado por autores como Baena y Tito Nelson Oviedo, que siendo mal parafraseados dieron origen a los estándares en lengua materna.

Hay que ser mucho más enfáticos en la necesidad de formar en Lingüística con miras su relación con la Educación y así con cada uno de sus campos de aplicación, para pensar que "los cursos de lingüística [en un pregrado en licenciatura] no deberían nunca gastarse en hacer un recuento del pensamiento de don fulano, ni del uno ni del otro, sino que deberían ser unos cursos organizados de tal modo que el muchacho aprenda a leer lingüística y si aprende a leer lingüística, lo demás ya él lo aprenderá solo" (Baena, 1996, p. 29).

\section{Referencias}

Baena, L. (1989). Funciones del lenguaje y enseñanza de la lengua. En lenguaje, 17.

Baena, L. (1989). Lingüística y significación. En Lenguaje, 17.

Barriga, F. \& Hernández, G. (2010). Estrategias docentes para un aprendizaje significativo. Una interpretación constructivista. México DF: Mc Graw Hill.

Cardona, E. \& Castillo, S. (2009). La enseñanza de la lectura académica: un objeto de formación docente. En Lectura y vida: Revista latinoamericana de lectura, 30 (1), 56-67. Recuperado de: http://dialnet.unirioja.es/ servlet/articulo?codigo $=2962144$

Castillo, D. (2009). ¿Son los profesores de lenguaje, investigadores del Pensamiento Crítico?. En Cuadernos de Lingüística Hispánica, (13), 129-142. Recuperado de: http://dialnet. unirioja.es/servlet/articulo?codigo $=3322190$

Corredor, J. \& Romero, C. (2009). Quiz de lingüística general: breve relato de una experiencia pedagógica en el aula. En Cuadernos de lingüística hispánica, (13), 91112. Recuperado de: http://dialnet.unirioja. es/servlet/articulo?codigo $=3322169$

Halladay, M. (1982). El lenguaje como semiótica social. México: Fondo de Cultura Económica

López, N. \& Puentes, A. (2011). Modernización curricular dela universidad Surcolombiana: integración e interdisciplinariedad. En Entornos, 24, 103-122.

Mahecha, M. (2008). Hacia una pedagogía de la lingüística- El Rol del lingüista. Paideia Surcolombiana, (13), 1-15.Recuperado de: http://www.paideiasurcolombiana.com/ edicion/numero-13_6

Mahecha, M. (2009). ¿Para qué enseñamos lingüística? Paideia Surcolombiana, (14), 21-33. Recuperado de: http://www. paideiasurcolombiana.com/edicion/ numero-14_1

Mahecha, M. (2012). Antología de lingüística cognitiva. Universidad Surcolombiana.

Marciales, G. (2003). Pensamiento crítico: diferencias en estudiantes universitarios en el tipo de creencias, estrategias e inferencias en la lectura crítica de textos. Universidad Complutense, Madrid, España.

Oviedo, T. (2002). Comunicación y educación. Lenguaje, 29-30. Recuperado de: http:// revistalenguaje.univalle.edu.co/index. php?seccion=REVISTA\&r evista=29-30 
Suárez, K. (2009). ¿Es la pedagogía la herramienta de la práctica docente?. En Paideia Surcolombiana, 14, 15-19. Recuperado de: http://www.paideiasurcolombiana.com/ edicion/numero-14_1 